ARTICLE

Received 15 Mar 2015 | Accepted 10 Aug 2015 | Published 15 Sep 2015

DOI: $10.1038 /$ ncomms 9318

\title{
Covalency of hydrogen bonds in liquid water can be probed by proton nuclear magnetic resonance experiments
}

\author{
Hossam Elgabarty ${ }^{1}$, Rustam Z. Khaliullin ${ }^{2,3} \&$ Thomas D. Kühne ${ }^{1,4}$
}

The concept of covalency is widely used to describe the nature of intermolecular bonds, to explain their spectroscopic features and to rationalize their chemical behaviour. Unfortunately, the degree of covalency of an intermolecular bond cannot be directly measured in an experiment. Here we established a simple quantitative relationship between the calculated covalency of hydrogen bonds in liquid water and the anisotropy of the proton magnetic shielding tensor that can be measured experimentally. This relationship enabled us to quantify the degree of covalency of hydrogen bonds in liquid water using the experimentally measured anisotropy. We estimated that the amount of electron density transferred between molecules is on the order of $10 \mathrm{~m} \bar{e}$ while the stabilization energy due to this charge transfer is $\sim 15 \mathrm{~kJ} \mathrm{~mol}^{-1}$. The physical insight into the fundamental nature of hydrogen bonding provided in this work will facilitate new studies of intermolecular bonding in a variety of molecular systems.

\footnotetext{
${ }^{1}$ Department of Chemistry, Dynamics of Condensed Matter, University of Paderborn, Warburger Street 100, D-33098 Paderborn, Germany. ${ }^{2}$ Department of Chemistry, University of Zurich, Winterthurerstrasse 190, CH-8057 Zurich, Switzerland. ${ }^{3}$ Department of Chemistry, McGill University, 801 Sherbrooke Str. West, Montreal, Quebec, Canada H3A OB8. ${ }^{4}$ Paderborn Center for Parallel Computing and Institute for Lightweight Design with Hybrid Systems, Warburger Street 100, D-33098 Paderborn, Germany. Correspondence and requests for materials should be addressed to T.D.K. (email: tdkuehne@mail.upb.de).
} 
$\mathrm{F}$ rom its early days, NMR spectroscopy has been instrumental in the study of liquid water ${ }^{1,2}$. The magnetic shielding tensor, which relates secondary induced electronic magnetic fields to an external static magnetic field, is at the heart of NMR spectroscopy. The shielding tensor is characterized by the three components in the principal axes frame $\sigma_{X} \leq \sigma_{Y} \leq \sigma_{Z}$ or, alternatively, by the isotropic part $\sigma_{\text {iso }}=\frac{1}{3}\left(\sigma_{X}+\sigma_{Y}+\sigma_{Z}\right)$, the anisotropy $\Delta=\sigma_{Z}-\sigma_{\text {iso }}$, and the asymmetry $\eta=\frac{1}{\Delta}\left(\sigma_{Y}-\sigma_{X}\right)^{3}$. However, because of the random orientation and motion of molecules in the liquid phase, only the isotropic part of the tensor can be directly measured by experiment. Nevertheless, even the isotropic ${ }^{1} \mathrm{H}$ shielding can provide valuable information: it has been long established that $\sigma_{\text {iso }}$ decreases on the formation of hydrogen bonds $(\mathrm{HBs})^{4-6}$.

A quantitative investigation between the other components of the shielding tensor and the $\mathrm{HB}$ geometry in liquid water ${ }^{7,8}$ and small cluster models $4,9,10$ has been made possible by advances in electronic structure theory ${ }^{11-16}$. Moreover, a recently reported elegant experimental approach has allowed the indirect measurement of the ${ }^{1} \mathrm{H}$ shielding anisotropy in liquid water via its contribution to spin relaxation rates ${ }^{17}$. Combined with first principles calculations, the new experimental data ${ }^{8}$ have underscored previous findings that some of the tensor components are more sensitive to $\mathrm{HB}$ interactions than the average $\sigma_{\text {iso }}$ (refs 4,18).

In this work, we reveal a quantitative relationship between the calculated components of the ${ }^{1} \mathrm{H}$ magnetic shielding tensor and the degree of covalency of HBs in liquid water ${ }^{19,20}$. The covalent component of intermolecular bonds is commonly defined as the strength of donor-acceptor orbital interactions between molecules ${ }^{21}$. Covalency, like many other individual fundamental mode of binding (for example, frozen electrostatics, polarization), cannot be measured, even in principle, by experiment; only the total strength of binding is measurable. Nevertheless, similar to various other unmeasurable auxiliary concepts (for example, wave function in quantum mechanics), covalency is a fundamental, theoretically well defined and physically meaningful quantity that is widely used by chemists to investigate the nature of intermolecular bonding. Furthermore, it is widely accepted that donor-acceptor interactions between molecules in liquid water affect its directly measurable structural, spectroscopic and chemical properties.

\section{Results}

Decomposition analysis and NMR shielding tensor. To quantify the strength of covalent interactions in a $\mathrm{HB}$, we employed the decomposition analysis for condensed phase systems based on absolutely localized molecular orbitals (ALMO DA) $)^{21,22}$ within Kohn-Sham density functional theory ${ }^{23}$. The ALMO DA makes this feat possible by first filtering out frozen electrostatic and polarization effects from the total many-body intermolecular binding energy, and then splitting the remaining covalent component into two-body terms, each corresponding to an individual HB. The ALMO approach allows to define two scales to measure the strength of covalent interactions: the amount

\begin{tabular}{|c|c|c|c|c|c|c|}
\hline & $\sigma_{\text {iso }}$ & $\Delta$ & $\eta$ & $\sigma_{X}$ & $\sigma_{Y}$ & $\sigma_{\boldsymbol{Z}}$ \\
\hline mean & 27.34 & 18.00 & 0.13 & 17.21 & 19.48 & 45.33 \\
\hline s.e. & 0.04 & 0.06 & 0.001 & 0.07 & 0.07 & 0.05 \\
\hline s.d. & 2.21 & 3.10 & 0.08 & 3.42 & 3.57 & 2.29 \\
\hline
\end{tabular}

of electron density transferred from the occupied orbitals on molecule $\mathrm{A}$ to the virtual orbitals on molecule $\mathrm{B}\left(\Delta Q_{\mathrm{A} \rightarrow \mathrm{B}}\right)$, and the energetic stabilization due to this charge transfer $\left(\Delta E_{\mathrm{A} \rightarrow \mathrm{B}}\right)$. These two-body terms are obtained self-consistently and include cooperativity effects, which are essential for a correct description of the HB network in liquid water ${ }^{24-27}$.

All ensemble averaged components of the NMR shielding tensor (Table 1), geometric and ALMO DA descriptors of HBs were calculated from density functional theory-based $a b$ initio molecular dynamics (MD) simulations performed to represent liquid water at ambient conditions (see Methods for details). The computed values are close to the experimentally measured absolute shielding values at $27^{\circ} \mathrm{C}\left(\sigma_{\text {iso }}=25.7\right.$ p.p.m. and $\Delta=18.3$ p.p.m., respectively $)^{17}$. We followed the common practice of denoting the average of $\sigma_{X}$ and $\sigma_{Y}$ components of the axially nearly symmetric NMR tensors $(\eta \approx 0)$ as the perpendicular shielding component $\sigma_{\perp}$. It is easy to see that $\sigma_{\perp}$ is directly related to the anisotropy: $\sigma_{\perp}=\sigma_{\text {iso }}-\frac{\Delta}{2}$.

Correlation between $\sigma$ components, HB covalency and geometry. Figure 1a,d show that $\sigma_{\perp}$ exhibits a striking correlation with the strength of electron density transfer as measured on both charge and energy scales. By comparison, the correlation between $\sigma_{\text {iso }}$ and the strength of charge transfer (Fig. 1c,f) is weaker, because $\sigma_{\text {iso }}$ is just the strongly correlated $\sigma_{\perp}$ component plus the noise from $\sigma_{Z}$ (Fig. 1b,e).

The presence of the scattered points in Fig. 1 that do not follow the general trend is a manifestation of the complex nature of the $\mathrm{HB}$ network, in which some hydrogen atoms do not form HBs and some interact strongly with more than one electron donor. Describing such configurations in detail is beyond the scope of the present work and will be presented elsewhere. Here we just would like to note that including or excluding the outliers does not change our statistical results, models or conclusions significantly (see Methods).

To obtain a more comprehensive view on $\mathrm{HBs}$ in liquid water we collected information on the correlation between electronic, NMR and geometric descriptors into a single matrix shown in Fig. 2. In addition to the key relationship established above, the correlation matrix clearly shows the well-known relationship between the $\mathrm{HB}$ length and $\sigma_{\text {iso. }}$. However, we found that the correlation of the $\mathrm{HB}$ length and $\sigma_{\perp}$ is stronger. As in the case of the electronic descriptors, the correlation between the HB length and $\sigma_{\text {iso }}$ is merely a manifestation of the underlying strong correlation with $\sigma_{\perp}$ plus a noisy contribution from $\sigma_{Z}$.

$\Delta Q_{\mathrm{A} \rightarrow \mathrm{B}}$ and $\Delta E_{\mathrm{A} \rightarrow \mathrm{B}}$ are also strongly correlated with the $\mathrm{HB}$ length, even though this correlation is somewhat weaker than that with $\sigma_{\perp}$ because the HB length alone is insufficient to characterize a $\mathrm{HB}$ (that is, other geometric descriptors, such as the $\mathrm{HB}$ angle, should be taken into account). We note that no correlations were found for the electron transfer terms where the water molecule bearing the shielding tensor acts as an electron donor. This shows that the ${ }^{1} \mathrm{H}$ shielding tensor is insensitive to electron transfer from the lone pairs of its own water molecule. There is no significant correlation of the $\mathrm{HB}$ angle with any individual shielding tensor component, except for a rather weak correlation with $\Delta$. The $\mathrm{HB}$ angle alone does not appear to influence electronic terms either, in agreement with the insensitivity of $\Delta Q_{\mathrm{A} \rightarrow \mathrm{B}}$ and $\Delta E_{\mathrm{A} \rightarrow \mathrm{B}}$ to intermolecular rotations in water dimer ${ }^{28}$.

In addition to studying the shape of the shielding ellipsoid, we also examined its orientation relative to the water molecule. As in previous studies $^{29}$, our simulations showed that the longest $Z$ axis of the tensor points along the covalent $\mathrm{O}-\mathrm{H}$ bond: the mean deviation angle between the longest axis and the covalent $\mathrm{O}-\mathrm{H}$ 


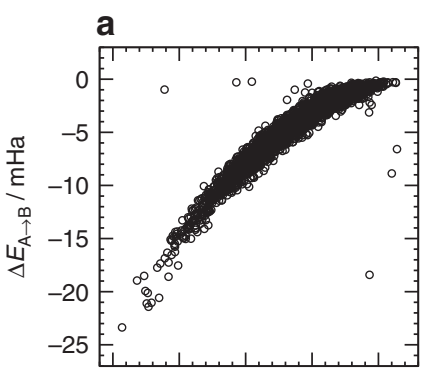

b
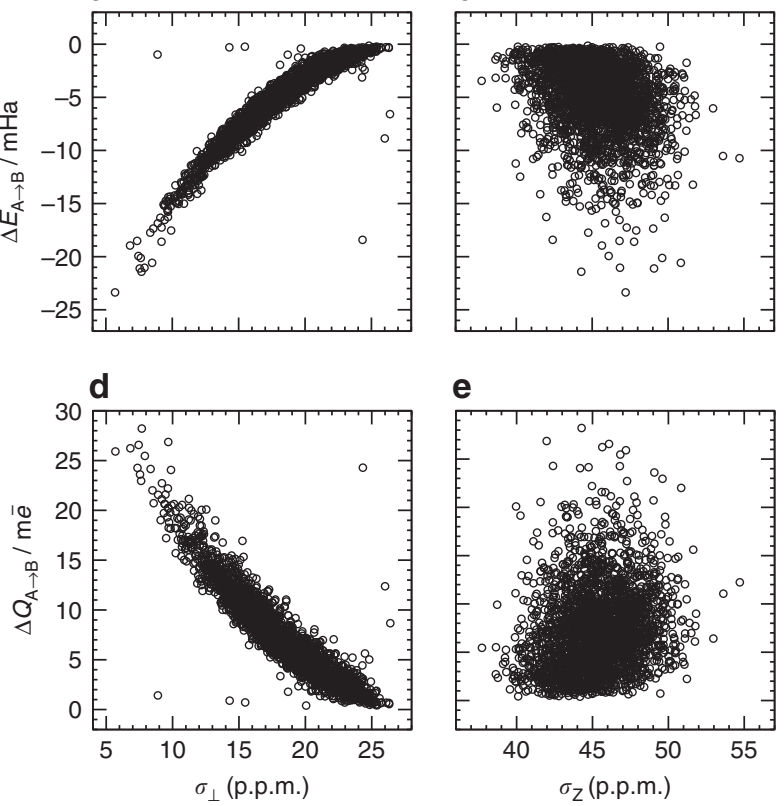

e

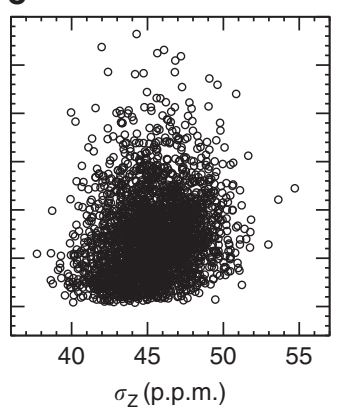

C

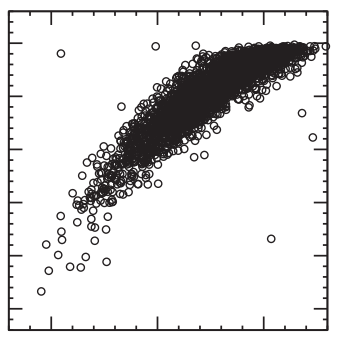

$\mathbf{f}$

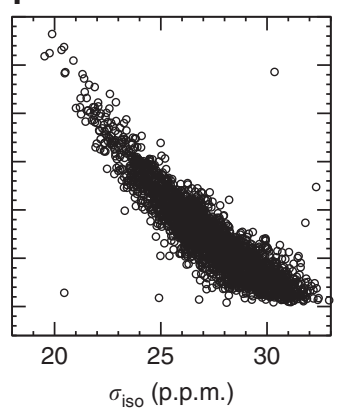

Figure 1 | Relationship between the electronic descriptors and NMR characteristics of HBs. $\Delta E_{\mathrm{A} \rightarrow \mathrm{B}}$ and $\Delta \mathrm{Q}_{\mathrm{A} \rightarrow \mathrm{B}}$ as a function of $\sigma_{\perp}, \sigma_{Z}$ and $\sigma_{\text {isol }}$ respectively.

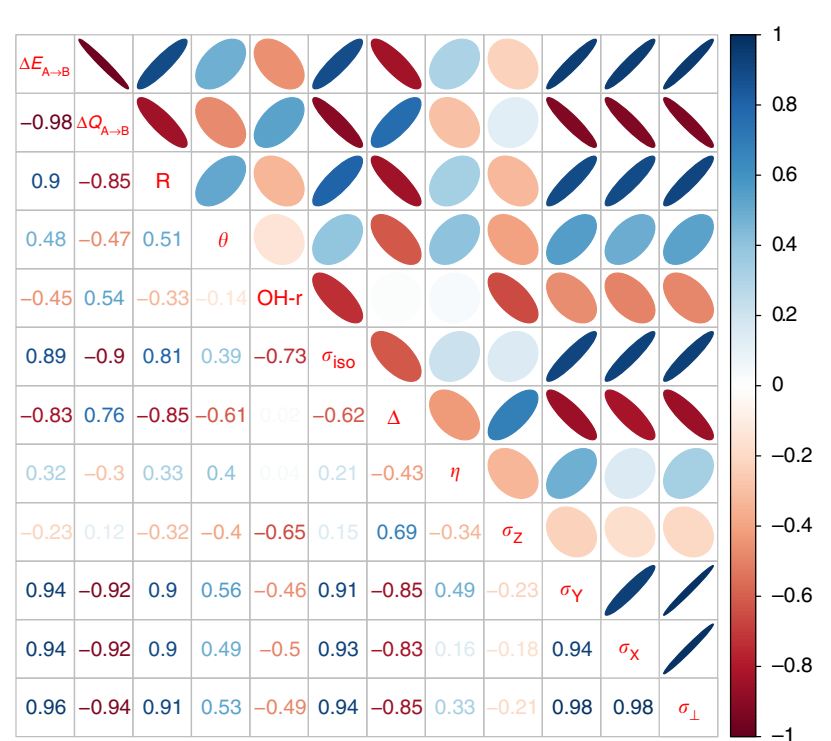<smiles>[2H]C1CCCOC1OCCO</smiles>

Figure 2 | Correlation matrix between the ${ }^{1} \mathrm{H}$ shielding tensor components and HB descriptors. The descriptors are shown on the main diagonal. The lower part of the matrix gives numerical values of the Pearson correlation coefficient $r$ between a pair of variables. The upper part above the diagonal presents a symbolic representation of the same correlation: ellipses represent different degrees of correlation ranging from $r=1$ (straight line) to $r=0$ (perfect circle). The geometric descriptors are illustrated on the water dimer at the bottom, where $\mathrm{OH}-\mathrm{r}$ is the covalent $\mathrm{O}-\mathrm{H}$ bond length and $\mathrm{R}$ the $\mathrm{HB}$ length, while $\theta$ is the $\mathrm{HB}$ angle.
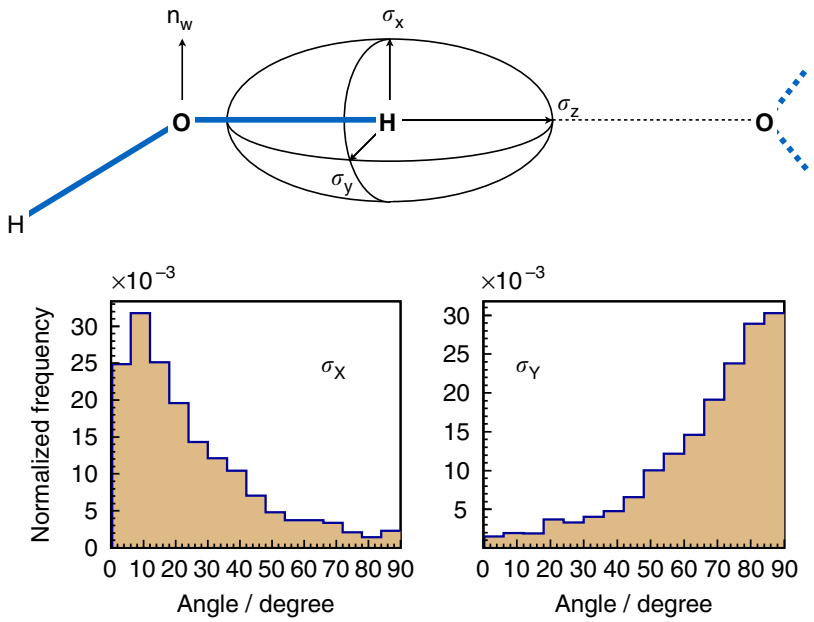

Figure 3 | Spatial orienation of the ${ }^{\mathbf{1}} \mathbf{H}$ shielding tensor relative to the $\mathbf{H B}$ geometry. The ${ }^{1} \mathrm{H}$ shielding tensor ellipsoid is rigidly fixed to the molecular frame of the HB donating water molecule. The longest axis of the ellipsoid $\left(\sigma_{Z}\right)$ always points along the HB. The angle between the normal to the water molecular plane $\left(\mathrm{n}_{\mathrm{w}}\right)$ and $\sigma_{X}$ and $\sigma_{Y}$ show that the former is parallel to $n_{w}$ while the latter is orthogonal to it.

bond is only $5 \pm 3^{\circ}$ (Fig. 3 , top). Moreover, despite the very low value of $\eta$, we find that the two short axes still exhibit a distinct spatial preference: the shortest $X$ axis is normal to the plane of the water molecule, whereas the intermediate $Y$ axis is coplanar with the molecule (Fig. 3, bottom). In other words, the shielding tensor is rigidly fixed to the water molecule. Previous reports have shown that the only non-zero off-diagonal elements of the shielding tensor are $\sigma_{y z}$ and $\sigma_{z y}$ in the Eckart frame $\mathrm{e}^{7,30}$. This is consistent with our finding, as the transformation from the principal axes frame to the Eckart frame is merely a rotation around the $X$ axis. 
Linear model that relates $\sigma_{\perp}$ to the covalency of HBs. The physical basis underlying the correlation between the electronic and NMR terms (Fig. 1) can be understood by considering the dependence of both of these quantities on the HB length $R$. We found that $\sigma_{x}$ and $\sigma_{y}$ decrease linearly with $R^{-3}$ over the entire HB length range in liquid water (see Supplementary Fig. 1). This strong negative correlation implies that the induced magnetic field from the electron donor molecule (a schematic depiction is given in Supplementary Fig. 2) is the major contributor to deshielding in the plane orthogonal to the $\mathrm{HB}$-it accounts for $88 \%$ of the variance in $\sigma_{\perp}$. The inverse cubic dependence also suggests that this field can be accurately represented as that of a magnetic dipole. That is, higher order terms in the multipole expansion (Supplementary Equation 1) are negligible. Thus, the $R^{-3}$ dependence of $\sigma_{\perp}$ enables one to write:

$$
\sigma_{\perp} \simeq \sigma_{\perp}^{\infty}-\alpha R^{-3}
$$

where $\sigma_{\perp}^{\infty}$ characterizes an HB-free water molecule, and has a value of 33.7 p.p.m. as obtained by linearly extrapolating $\sigma_{\perp}$ to $R^{-3}=0$. The strength of donor-acceptor interactions between molecules decreases with the tails of the orbitals, that is, exponentially with distance ${ }^{21}$

$$
\Delta Q_{A \rightarrow B} \simeq \lambda_{Q} \exp \left(-\beta_{Q} R\right) .
$$

The combination of the two equations predicts the following relationship between the charge-transfer term and perpendicular component of the NMR shielding tensor:

$$
\log \left[\frac{\Delta Q_{A \rightarrow B}}{\lambda_{Q}}\right] \simeq \gamma_{Q}\left(\sigma_{\perp}^{\infty}-\sigma_{\perp}\right)^{-1 / 3},
$$

where $\gamma_{Q}=-\beta_{Q} \alpha^{1 / 3}$ is the dimensionless proportionality constant. A similar relationship can be written for the energy scale with $\beta_{E}, \gamma_{E}, \lambda_{E}$ as parameters. Indeed, plotting both sides of equation (3) reveals clear linear trends (Fig. 4) with a Pearson coefficient of -0.94 for $\Delta E_{\mathrm{A} \rightarrow \mathrm{B}}$ and -0.92 for $\Delta Q_{\mathrm{A} \rightarrow \mathrm{B}}$. The values of the parameters that represent the best fit of the simulation data are $\lambda_{Q}=270.6 \mathrm{~m} \bar{e}, \lambda_{E}=579.4 \mathrm{mHa}$, $\gamma_{Q}=-19.86$ and $\gamma_{E}=-24.07$, respectively. It is worth noting that the $\lambda$ coefficients should not be interpreted as the limiting values for $R \rightarrow 0$, but rather as statistical coefficients that best fit the linear trend over its limited range of validity.

While $\sigma_{\perp}$ shows the strong $R^{-3}$ dependence, the weak correlation between $\sigma_{Z}$ and $R^{-3}$ (Supplementary Fig. 1) indicates that the change in $\sigma_{Z}$ results from the factors other than the dipolar field of the electron donor molecule. We found that a satisfactory regression model capable of predicting the behaviour of $\sigma_{Z}$ should include at least three predictor variables: $R^{-3}$, the $\mathrm{HB}$ angle $\theta$ and the covalent $\mathrm{O}-\mathrm{H}$ bond length $\mathrm{OH}-\mathrm{r}$. The standardized regression coefficients of this model (Supplementary a

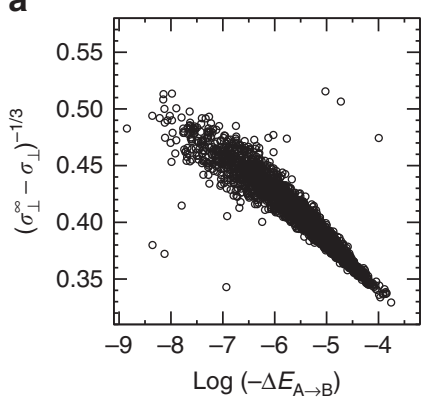

b

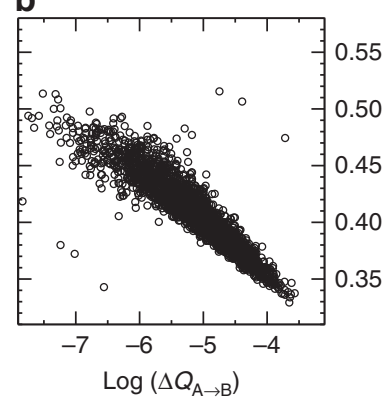

Figure 4 | The derived linear model relating $\sigma_{\perp}$ to the electronic descriptors as obtained by ALMO DA. Dependence between $\left(\sigma_{\perp}^{\infty}-\sigma_{\perp}\right)^{-1 / 3}$ and: $(\mathbf{a}) \log \left(-\Delta E_{\mathrm{A} \rightarrow \mathrm{B}}\right),(\mathbf{b}) \log \left(\Delta \mathrm{Q}_{\mathrm{A} \rightarrow \mathrm{B}}\right)$.
Table 1) show that $R^{-3}$ and $\mathrm{OH}-\mathrm{r}$ give opposite contributions to $\sigma_{Z}$ largely cancelling each other out.

The established quantitative dependence between $\sigma_{\perp}$ and the strength of intermolecular donor-acceptor bonding has one important implication: since $\sigma_{\perp}$ can now be measured experimentally in liquids ${ }^{17}$, our model represents an indirect method for probing the covalent component of HBs. Table 2 presents our estimates of $\Delta E_{\mathrm{A} \rightarrow \mathrm{B}}$ and $\Delta Q_{\mathrm{A} \rightarrow \mathrm{B}}$ in liquid water based on a linear approximation to the regression model established here and the $\sigma_{\perp}$ measured experimentally at different temperatures ${ }^{17}$ (Supplementary Note 1 for full analysis). As expected, the strength of the covalent component in HBs decreases with increasing temperature as more water molecules form distorted or even broken HBs with their neighbours.

We would like to emphasize that, unlike population analysis methods (for example, Mulliken, Löwdin), which partition the total electron density between molecules, the ALMO approach measures the reorganization of electron clouds on the formation of a $\mathrm{HB}^{31}$. This conceptual advantage of the ALMO method leads to the remarkable result that the fractional electron transfer $\Delta Q_{\mathrm{A} \rightarrow \mathrm{B}}$ between water molecules in the liquid phase is only a few milli-electrons (Table 2). The reorganization of charge has a well-defined energy given by the $\Delta E_{\mathrm{A} \rightarrow \mathrm{B}}$ term. Whereas it may seem unusual that so little charge transfer $(7-10 \mathrm{~m} \bar{e})$ can stabilize a $\mathrm{HB}$ by $12-18 \mathrm{~kJ} \mathrm{~mol}^{-1}$ (equivalent to $19 \mathrm{eV}$ per one transferred electron), it is consistent with simple theoretical estimates. Perturbation theory shows that the transfer energy per electron is equal to the energy gap between donating and accepting orbitals $^{28}$. Since the energy gap between the most important donating and accepting orbitals on two molecules lies between 10 and $40 \mathrm{eV}$ (virtual orbitals form almost a continuum of states), a value of $19 \mathrm{eV}$ for the effective donor-acceptor gap is entirely reasonable.

To put the estimates of $\Delta Q_{\mathrm{A} \rightarrow \mathrm{B}}$ into a context, we calculated that $4 \mathrm{~m} \bar{e}$ is transferred between molecules in the water dimer if the two molecules are at their equilibrium separation of $2.0 \AA$. However, $\Delta Q_{\mathrm{A} \rightarrow \mathrm{B}}$ becomes $7.6 \mathrm{~m} \bar{e}$ (that is, significantly closer to the value obtained for the ambient liquid) if the molecules in the dimer are separated only by $1.77 \AA$-the typical length of a HB in the liquid phase ${ }^{27}$.

It is important to note that the timescale of fluctuations in $\Delta Q_{\mathrm{A} \rightarrow \mathrm{B}}$ and $\Delta E_{\mathrm{A} \rightarrow \mathrm{B}}$ is the same as the timescale of intermolecular vibrations-hundreds of femtoseconds ${ }^{22,32-34}$, which is several orders of magnitude faster than the typical timescale of NMR spectroscopy. This implies that NMR measurements of covalency are capable of yielding only time-averaged values of $\Delta Q_{\mathrm{A} \rightarrow \mathrm{B}}$ and $\Delta E_{\mathrm{A} \rightarrow \mathrm{B}}$.

\section{Discussion}

To summarize, we established a simple quantitative relationship between the perpendicular component $\sigma_{\perp}$ of the axially nearly symmetric ${ }^{1} \mathrm{H}$ magnetic shielding tensor and the degree of covalency of HBs in liquid water. Covalency was defined as the amount of electron density transfer between water molecules and

Table 2 | Estimated average values for $\Delta E_{A \rightarrow B}$ and $\Delta Q_{A \rightarrow B}$ in liquid water at different temperatures.

\begin{tabular}{lccc}
$\mathbf{T .} /{ }^{\circ} \mathbf{C}$ & $\boldsymbol{\sigma}_{\perp}$ (p.p.m.) & $\boldsymbol{\Delta} \mathbf{Q}_{\mathbf{A} \rightarrow \mathrm{B}} / \mathbf{m} \overline{\mathbf{e}}$ & $\boldsymbol{\Delta} \boldsymbol{E}_{\mathbf{A} \rightarrow \mathbf{B}} / \mathbf{m H a}$ \\
\hline 80 & 17.9 & $7.09(7.02,7.17)$ & $-4.68(-4.63,-4.73)$ \\
27 & 16.6 & $8.71(8.61,8.82)$ & $-6.01(-5.93,-6.08)$ \\
0 & 15.9 & $9.65(9.53,9.78)$ & $-6.80(-6.71,-6.89)$
\end{tabular}

The estimates are based on the experimentally determined $\sigma_{\perp}$ (ref. 17) and the regression model given by Equation (3). Brackets represent the $95 \%$ C.I. of the regression model. 
the stabilization energy associated with it. The physical origin of this relationship is the field induced almost entirely by the magnetic dipole located on the neighbouring water molecule involved in the HB. The major implication of our findings is that this relationship provides the calibration necessary to quantify the covalency of HBs experimentally.

Recent advancements in measuring $\sigma_{\perp}$ for liquid water ${ }^{17}$ enabled us to estimate that the average amount of charge transferred between the molecules on the formation of an average $\mathrm{HB}$ is on the order of $10 \mathrm{~m} \bar{e}$, while the corresponding stabilization energy is estimated to be $15 \mathrm{~kJ} \mathrm{~mol}^{-1}$. From the practical perspective, using $\sigma_{\perp}$ rather than $\sigma_{X}$ or $\sigma_{Y}$ offers an experimental advantage because $\sigma_{\perp}$ can be determined as a linear combination of $\sigma_{\text {iso }}$ and $\Delta$ and it is technically easier to measure the latter.

In contrast to $\sigma_{\perp}$, the $\sigma_{Z}$ component of the shielding tensor exhibits a complex dependence on the local environment around the proton. Its fluctuations cannot be explained only by the magnitude of the induced magnetic fields originating at the proton-accepting water molecule. Therefore, although it is trivial to measure $\sigma_{\text {iso }}$ experimentally, the noisy contribution of $\sigma_{Z}$ makes it unsuitable for predicting covalency.

\section{Methods}

Second generation Car-Parrinello $\boldsymbol{a b}$ initio MD simulations. A $b$ initio MD simulations of a periodic cubic cell with 128 light water molecules were performed at constant temperature ( $330 \mathrm{~K}$ to mimic nuclear quantum effects in liquid water at ambient conditions $\left.{ }^{35}\right)$ and density $\left(0.9966 \mathrm{~g} \mathrm{~cm}^{-3}\right)$ using the second generation Car-Parrinello method ${ }^{36,37}$. In this approach, the fictitious Newtonian dynamics of the original Car-Parrinello scheme $e^{38}$ is replaced with an improved coupled electron-ion dynamics that keeps electrons close to the instantaneous electronic ground state without defining a fictitious mass parameter. The superior efficiency originates from the fact that the iterative wave function optimization is fully bypassed. Thus, not even a single diagonalization step is required, while at the same time allowing for integration time steps up to the ionic resonance limit.

The energy and forces were computed using the mixed Gaussian-plane waves approach $^{39}$, where the Kohn-Sham orbitals were represented by an accurate triple- $\zeta$ basis set with two sets of polarization functions (TZV2P) ${ }^{40}$, while planewaves with cutoff of $400 \mathrm{Ry}$ were used to represent the charge density. The BLYP exchange-correlation functional plus a damped interatomic potential to account for van der Waals interactions ${ }^{41}$ was employed. Previous works have shown that this set-up provides a realistic description of many important structural, dynamical and spectroscopic characteristics of liquid water, including the partial pair correlation functions, self-diffusion and viscosity coefficients, HB lifetime, vibrational spectra and magnetic shielding tensor components $14,15,27,42,43$. The system has been equilibrated at constant temperature and density for $30 \mathrm{ps}$ before ten decorrelated snapshots separated by 1 ps were extracted.

Computational analysis. The ALMO DA was performed for each snapshot using the same computational set-up as in ref. 22. The magnetic shielding tensors were computed using density functional perturbation theory ${ }^{14,44,45}$ that is based on the all-electron GAPW method ${ }^{46,47}$ and IGLO-III basis set ${ }^{48}$. All computations were performed using the QUICKSTEP and ALMO modules of the CP2K suite of programmes ${ }^{49}$. To keep our results consistent, we performed sampling over all protons including the outliers that are clearly visible in Figs 1 and 4. The only exception is the analysis presented in Fig. 2 (supported by Supplementary Fig. 1 and Supplementary Table 1). The data presented in Fig. 2 requires computing geometric characteristics of HBs. These characteristics can be computed only for well defined HBs. To define a HB, we used a geometric criterion that was derived from the two-dimensional ( $R$ versus $\theta$ ) potential of mean force ${ }^{43,50}$.

\section{References}

1. Bloembergen, N., Purcell, E. \& Pound, R. Relaxation effects in nuclear magnetic resonance absorption. Phys. Rev. 73, 679-712 (1948).

2. Simpson, J. \& Carr, H. Diffusion and nuclear spin relaxation in water. Phys. Rev. 111, 1201-1202 (1958).

3. Harris, R. K. et al. Further conventions for NMR shielding and chemical shifts (IUPAC Recommendations 2008). Magn. Reson. Chem. 46, 582-598 (2008).

4. Ditchfield, R. Theoretical studies of magnetic shielding in $\mathrm{H} 2 \mathrm{O}$ and $(\mathrm{H} 2 \mathrm{O}) 2$ J. Chem. Phys. 65, 3123-3133 (1976).

5. Konrat, R., Tollinger, M., Kontaxis, G. \& Kräutler, B. NMR techniques to study hydrogen bonding in aqueous solution. Monatsh. Chem. 130, 961-982 (1999).

6. Grzesiek, S. \& Becker, E. D. Hydrogen Bonding. eMagRes. (2011).
7. Pennanen, T. S. et al. Nuclear magnetic shielding and quadrupole coupling tensors in liquid water: a combined molecular dynamics simulation and quantum chemical study. J. Am. Chem. Soc. 126, 11093-11102 (2004).

8. Modig, K., Pfrommer, B. \& Halle, B. Temperature-Dependent hydrogen-bond geometry in liquid water. Phys. Rev. Lett. 90, 075502 (2003).

9. Chesnut, D. \& Rusiloski, B. A study of NMR chemical shielding in water clusters derived from molecular dynamics simulations. J. Mol. Struct. 314, 19-30 (1994).

10. Limbach, H.-H. et al. OHO hydrogen and bond geometries and NMR chemical and shifts: from equilibrium structures to geometric H/D isotope effects, with applications for water, protonated water, and compressed ice. Isr. J. Chem. 49, 199-216 (2009)

11. Gauss, J. Calculation of NMR chemical shifts at second-order many-body perturbation theory using gauge-including atomic orbitals. Chem. Phys. Lett. 191, 614 (1992).

12. Gauss, J. Effects of electron correlation in the calculation of nuclear magnetic resonance chemical shifts. J. Chem. Phys. 99, 3629 (1993).

13. Pickard, C. \& Mauri, F. All-electron magnetic response with pseudopotentials: NMR chemical shifts. Phys. Rev. B 63, 1-13 (2001).

14. Sebastiani, D. \& Parrinello, M. A new ab Initio approach for NMR chemical shifts in periodic systems. J. Phys. Chem. A 105, 1951-1958 (2001)

15. Sebastiani, D. \& Parrinello, M. Ab-initio study of NMR chemical shifts of water under normal and supercritical conditions. Chem. Phys. Chem. 3, 675-679 (2002).

16. Ochsenfeld, C., Kussmann, J. \& Koziol, F. Ab Initio NMR Spectra for molecular systems with a thousand and more atoms: A linear-scaling method. Angew. Chem. Int. Ed. 43, 4485-4489 (2004).

17. Modig, K. \& Halle, B. Proton magnetic shielding tensor in liquid water. J. Am. Chem. Soc. 124, 12031-12041 (2002).

18. Saitô, H., Ando, I. \& Ramamoorthy, A. Chemical shift tensor - the heart of NMR: Insights into biological aspects of proteins. Prog. Nucl. Magn. Reson. Spectrosc. 57, 181-228 (2010).

19. Scheiner, S. Hydrogen bonding. A theoretical perspective (Oxford University Press, 1997).

20. Grabowski, S. J. What is the covalency of hydrogen bonding? Chem. Rev. 111, 2597-2625 (2011).

21. Khaliullin, R. Z., Cobar, E. A., Lochan, R. C., Bell, A. T. \& Head-Gordon, M. Unravelling the origin of intermolecular interactions using absolutely localized molecular orbitals. J. Phys. Chem. A 111, 8753-8765 (2007).

22. Khaliullin, R. Z. \& Kühne, T. D. Microscopic properties of liquid water from combined ab initio molecular dynamics and energy decomposition studies. Phys. Chem. Chem. Phys. 15, 15746-15766 (2013).

23. Kohn, W. Nobel lecture: electronic structure of matter - wave functions and density functionals. Rev. Mod. Phys. 71, 1253-1266 (1999).

24. Frank, H. S. Covalency in the Hydrogen bond and the properties of water and ice. Proc. Roy. Soc. A247, 481-492 (1958).

25. Luck, W. A. P. The importance of cooperativity for the properties of liquid water. J. Mol. Struct. 448, 131 (1998).

26. Glendening, E. D. Natural energy decomposition analysis: Extension to density functional methods and analysis of cooperative effects in water clusters. J. Phys. Chem. A 109, 11936-11940 (2005).

27. Kühne, T. D., Krack, M. \& Parrinello, M. Static and Dynamical properties of liquid water from first principles by a novel Car-Parrinello-like approach. J. Chem. Theory Comput. 5, 235-241 (2009).

28. Khaliullin, R. Z., Bell, A. T. \& Head-Gordon, M. Electron donation in the water-water hydrogen bond. Chem. Eur. J. 15, 851-855 (2009).

29. Pfrommer, B. G., Mauri, F. \& Louie, S. G. NMR chemical shifts of ice and liquid water: the effects of condensation. J. Am. Chem. Soc. 122, 123-129 (2000).

30. Vaara, J., Lounila, J., Ruud, K. \& Helgaker, T. Rovibrational effects, temperature dependence, and isotope effects on the nuclear shielding tensors of water: a new 17 O absolute shielding scale. J. Chem. Phys. 109, 8388-8397 (1998).

31. Khaliullin, R. Z., Bell, A. T. \& Head-Gordon, M. Analysis of charge transfer effects in molecular complexes based on absolutely localized molecular orbitals J. Chem. Phys. 128, 184112 (2008)

32. Fecko, C. J. Ultrafast hydrogen-bond dynamics in the infrared spectroscopy of water. Science 301, 1698-1702 (2003)

33. Kühne, T. D. \& Khaliullin, R. Z. Electronic signature of the instantaneous asymmetry in the first coordination shell of liquid water. Nat. Commun. 4, 1450 (2013).

34. Kühne, T. D. \& Khaliullin, R. Z. The nature of the asymmetry in the hydrogen-bond networks of hexagonal ice and liquid water. J. Am. Chem. Soc. 136, 3395-3399 (2014)

35. Morrone, J. \& Car, R. Nuclear quantum effects in water. Phys. Rev. Lett. 101, 017801 (2008).

36. Kühne, T. D., Krack, M., Mohamed, F. R. \& Parrinello, M. Efficient and accurate Car-Parrinello-like approach to Born- Oppenheimer molecular dynamics. Phys. Rev. Lett. 98, 0664501 (2007).

37. Kühne, T. D. Second generation Car-Parrinello molecular dynamics. Wiley Interdiscip. Rev. Comput. Mol. Sci. 4, 391 (2014). 
38. Car, R. \& Parrinello, M. Unified approach for molecular dynamics and densityfunctional theory. Phys. Rev. Lett. 55, 2471-2474 (1985).

39. Lippert, G., Hutter, J. \& Parrinello, M. A hybrid Gaussian and plane wave density functional scheme. Mol. Phys. 92, 477-488 (1997).

40. VandeVondele, J. \& Hutter, J. Gaussian basis sets for accurate calculations on molecular systems in gas and condensed phases. J. Chem. Phys. 127, 114105 (2007).

41. Grimme, S. Semiempirical GGA-type density functional constructed with a long-range dispersion correction. J. Comput. Chem. 27, 1787-1799 (2006).

42. Schmidt, J. et al. Isobaric-isothermal molecular dynamics simulations utilizing density functional theory: an assessment of the structure and density of water at near-ambient conditions. J. Phys. Chem. B 113, 11959-11964 (2009).

43. Kühne, T. D., Pascal, T. A., Kaxiras, E. \& Jung, Y. New Insights into the structure of the vapor/water interface from large-scale first-principles simulations. J. Phys. Chem. Lett. 2, 105-113 (2011).

44. Putrino, A., Sebastiani, D. \& Parrinello, M. Generalized variational density functional perturbation theory. J. Chem. Phys. 113, 7102 (2000).

45. Weber, V. et al. Magnetic linear response properties calculations with the Gaussian and augmented-plane-wave method. J. Chem. Phys. 131, 014106 (2009).

46. Lippert, G., Hutter, J. \& Parrinello, M. The Gaussian and augmented-planewave density functional method for ab initio molecular dynamics simulations. Theor. Chem. Acc. 103, 124-140 (1999).

47. Krack, M. \& Parrinello, M. All-electron ab-initio molecular dynamics. Phys. Chem. Chem. Phys. 2, 2105-2112 (2000).

48. Kutzelnigg, W., Fleischer, U. \& Schindler, M. NMR Basic Principles and Progress Vol. 23, 165-262 (Springer, 1991).

49. VandeVondele, J. et al. Quickstep: fast and accurate density functional calculations using a mixed Gaussian and plane waves approach. Comput. Phys. Commun. 167, 103-128 (2005).

50. Kumar, R., Schmidt, J. R. \& Skinner, J. L. Hydrogen bonding definitions and dynamics in liquid water. J. Chem. Phys. 126, 204107 (2007).

\section{Acknowledgements}

R.Z.K. is grateful for financial support from the Swiss National Science Foundation; T.D.K. kindly acknowledges the Gauss Center for Supercomputing (GCS) for providing computing time through the John von Neumann Institute for Computing (NIC) on the GCS share of the supercomputer JUQUEEN at the Jülich Supercomputing Centre (JSC).

\section{Author contributions}

H.E. and T.D.K. designed the research, H.E. and R.Z.K. performed the numerical calculations, R.Z.K. and T.D.K. developed and implemented the employed methodologies and computer codes, T.D.K. supervised the project. All authors contributed equally to writing the manuscript.

\section{Additional information}

Supplementary Information accompanies this paper at http://www.nature.com/ naturecommunications

Competing financial interests: The authors declare no competing financial interests.

Reprints and permission information is available online at http://npg.nature.com/ reprintsandpermissions/

How to cite this article: Elgabarty, H. et al. Covalency of hydrogen bonds in liquid water can be probed by proton nuclear magnetic resonance experiments. Nat. Commun. 6:8318 doi: $10.1038 /$ ncomms 9318 (2015)

(c) (i) This work is licensed under a Creative Commons Attribution 4.0 International License. The images or other third party material in this article are included in the article's Creative Commons license, unless indicated otherwise in the credit line; if the material is not included under the Creative Commons license, users will need to obtain permission from the license holder to reproduce the material. To view a copy of this license, visit http://creativecommons.org/licenses/by/4.0/ 